\title{
Corn Hybrid Response to Water Management Practices on Claypan Soil
}

\author{
Kelly A. Nelson ${ }^{1,2}$ and Randall L. Smoot ${ }^{2}$ \\ ${ }^{1}$ Division of Plant Sciences, University of Missouri, Novelty, MO 63460, USA \\ ${ }^{2}$ Greenley Research Center, University of Missouri, P.O. Box 126, Novelty, MO 63460, USA
}

Correspondence should be addressed to Kelly A. Nelson, nelsonke@missouri.edu

Received 19 January 2012; Revised 17 February 2012; Accepted 4 March 2012

Academic Editor: Bernd Lennartz

Copyright ( 12012 K. A. Nelson and R. L. Smoot. This is an open access article distributed under the Creative Commons Attribution License, which permits unrestricted use, distribution, and reproduction in any medium, provided the original work is properly cited.

\begin{abstract}
A study evaluated corn (Zea mays L.) hybrids (Asgrow785, DKC61-73, DKC63-42, LG2642, and Kruger2114) and water management systems (nondrained, nonirrigated (NDNI); drained, nonirrigated (DNI) with subsurface drain tiles 6.1 and $12.2 \mathrm{~m}$ apart; drained plus subirrigated (DSI) with tiles 6.1 and $12.2 \mathrm{~m}$ apart; nondrained, overhead irrigated (NDOHI)) on yields, plant population, and grain quality from 2008 to 2010. Precipitation during this study was 36 to $283 \mathrm{~mm}$ above the past decade. Planting date was delayed $18 \mathrm{~d}$ in the nondrained control in 2009, and additional delayed planting controls were included this year. Grain yields were similar in the 6.1- and $12.2 \mathrm{~m}$-spaced DNI and DSI systems in 2008 and 2010, but plant population increased $74 \%$ and yields were $3.1 \mathrm{Mg} \mathrm{ha}^{-1}$ greater with DSI at a $6.1 \mathrm{~m}$ spacing compared to $12.2 \mathrm{~m}$ in 2009 . At a $6.1 \mathrm{~m}$ spacing, DNI or DSI increased yield 1.1 to $6.6 \mathrm{Mg} \mathrm{ha}^{-1}$ (10 to over $50 \%$ ) compared to NDNI or NDOHI soil. High yielding hybrids achieved similar yields with DNI, while NDNI DKC63-42 had 1.2 $\mathrm{Mg} \mathrm{ha}^{-1}$ greater yields compared to DKC61-73. A $6.1 \mathrm{~m}$ spacing for DNI claypan soils is recommended for high yielding corn production.
\end{abstract}

\section{Introduction}

Within-season climate variability is a primary factor affecting corn yields in Missouri [1]. Although Midwestern farmers have been planting up to two weeks earlier than in the 1980s [2], recommendations for initiating planting continue to be based on field conditions and soil temperature [3]. Soils that are cool and wet can delay planting. Adequate soil drainage helps soils dry and warm quickly. The distribution of rainfall in upstate Missouri generally peaks in mid-April to midMay, with periods of drought and little water available to plants in late June, July, and early August [4-7]. Drought conditions during July and August are usually yield limiting in claypan soils, due to their low water-holding capacity $[1,4,8]$. However, these soils' poor drainage may contribute to excessive yield loss, due to stand loss, fertilizer loss, and poor root development $[4,6,8-10]$.

Several studies have evaluated corn response to drainage water management systems [11-14], interactions between drainage and nitrogen management [15-17], and the impacts of drainage on water quality [18-21]. Other studies have evaluated the effects of drainage and overhead irrigation on yield response of corn in a claypan soil in Southern Illinois [22-24]. Corn yields synergistically increased $4.8 \mathrm{Mg} \mathrm{ha}^{-1}$ with overhead irrigation and subsurface drainage [24]. In dry years, drainage plus subirrigation increased 3-year average yields $23 \%$ over drainage only in dry years [25] and conserved water compared to overhead irrigation systems [6]. In wet years, these conditions produced limited yield increases $[6,25]$. Soybean cultivar yield response to drainage plus subirrigation systems has been reported in Ohio [26] and Missouri [27]. But no known research has evaluated the interaction between corn hybrids and drainage water management systems at different drain tile spacings. Subsurface drains in claypan soils at $15-\mathrm{m}$ spacings were reported to remove from 41 to $47 \%$ of the excess rainfall [23]. Simulations in a claypan soil indicated the need for 6-m drain tile lateral spacing for subirrigation, though the 


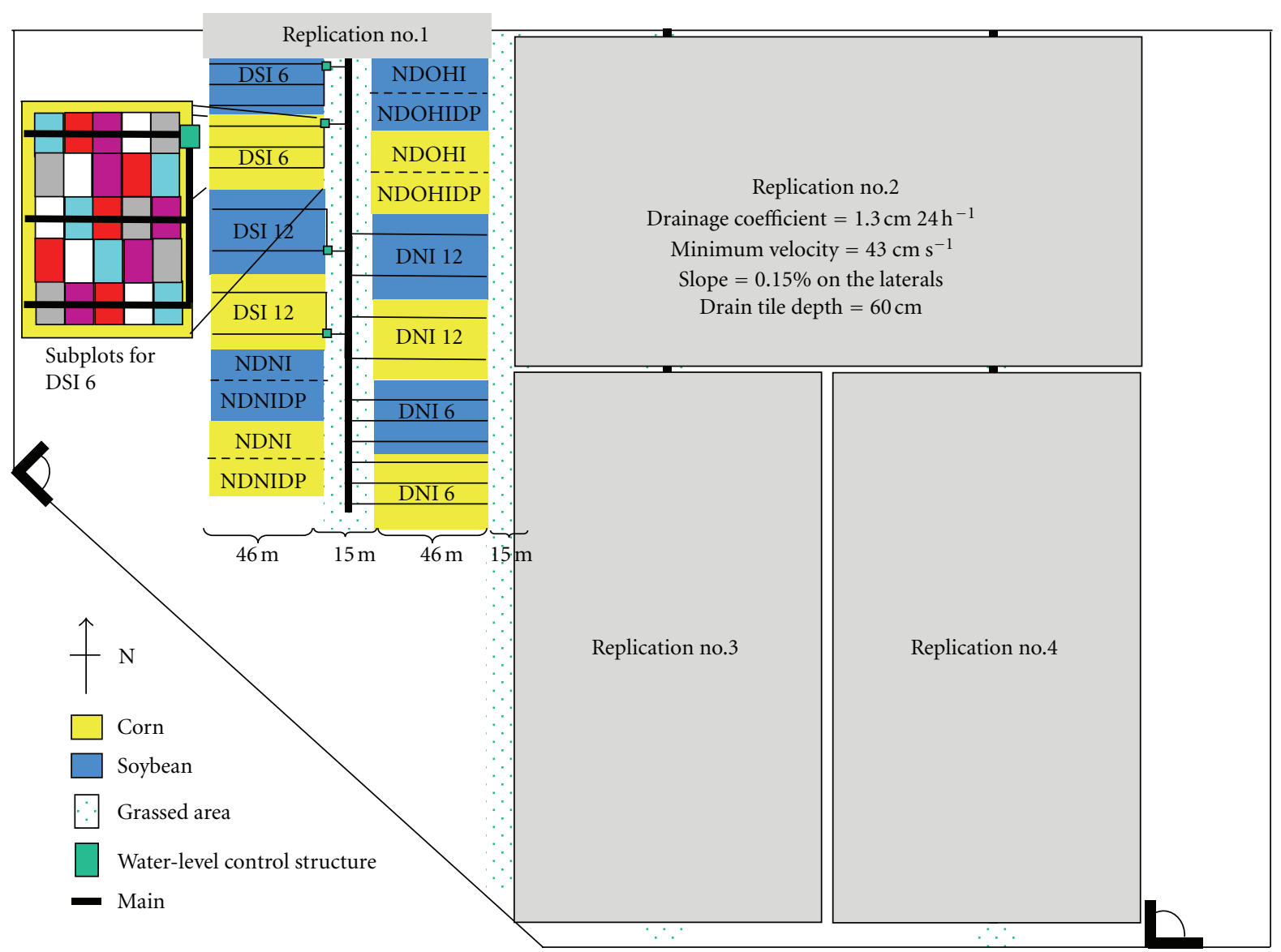

Figure 1: Split-plot design at the MUDS site. A representative subplot randomization above and between tile lines was represented by the insert for drainage plus subirrigation (DSI 6) at a $6.1 \mathrm{~m}$ spacing. The boxes with different colors represent five different hybrids randomly planted in 3 by $9.1 \mathrm{~m}$ plots above and between the tile lines. In 2009, the nondrained, nonirrigated (NDNI) and nondrained, overhead irrigated (NDOHI) controls were split to include a delayed planting (DP) treatment to avoid confounding planting dates between systems. Corn plots in 2008 and 2010 were rotated to soybean, and soybean plots were rotated into corn in 2009.

system's drainage component limited the lateral spacing [28]. However, little verification research has been conducted in corn [6], especially during extremely wet years. This research evaluated corn yield, plant population, and grain quality response to drainage only or drainage plus subirrigation at 6.1 and $12.2 \mathrm{~m}$ spacings and high yielding hybrids.

\section{Materials and Methods}

This study used drainage and irrigation plots at MU's Drainage and Subirrigation (MUDS) site located near the University of Missouri Lee Greenley Jr. Memorial Research Center on the Ross Jones farm $\left(39^{\circ} 56^{\prime} \mathrm{N}, 92^{\circ} 3^{\prime} \mathrm{W}\right)$ near Bethel, MO, from 2008 to 2010 . The site has been previously described $[6,27,29,30]$. The soil was a Putnam silt loam (fine, smectitic, mesic Vertic Albaqaulfs) with poor drainage, slope of less than $1 \%$, and a Bt horizon with a claypan at a depth of approximately $51 \mathrm{~cm}$. The drainage and drainageplus-subirrigation systems were installed in 2001. Drain tiles ( $7.6 \mathrm{~cm}$ diameter) were installed $60 \mathrm{~cm}$ deep at $0.15 \%$ slope with a minimum flow velocity of $43 \mathrm{~cm} \mathrm{sec}^{-1}$ and a drainage coefficient of $1.3 \mathrm{~cm} 24 \mathrm{~h}^{-1}$.
The experiment was arranged in a split-plot design with four replications (Figure 1). The main plot was water management system (nondrained, nonirrigated (NDNI); nondrained, overhead irrigated (NDOHI); drained, nonirrigated at $6.1 \mathrm{~m}$ drain spacing (DNI 6); drained, nonirrigated at $12.2 \mathrm{~m}$ drain spacing (DNI 12); drained, subirrigated at $6.1 \mathrm{~m}$ drain spacing (DSI 6); drained, subirrigated at $12.2 \mathrm{~m}$ drain spacing (DSI 12)), and subplots were hybrids (Asgrow785, DKC61-73, DKC63-42, LG2642, and Kruger2114). Subplots were randomized above and between the drain tiles in 3 by $9.1 \mathrm{~m}$ plots. The total number of plots was 30 in 2008 and 2010. Soil conditions in 2009 were favorable for planting in the DNI and DSI treatments on 24 Apr. Soil conditions were poor for tillage and planting in the nondrained control, but half of the plot area was planted to avoid confounding results due to a delayed planting date. The delayed planting control treatments were denoted as nondrained, nonirrigated, and delayed planting (NDNIDP) and overhead irrigated, nondrained, and delayed planting (NDOHIDP), which increased the total number of plots to 40. Rainfall following planting was intense and extensive (Figure 2(b)). As a result, planting of the NDNIDP 


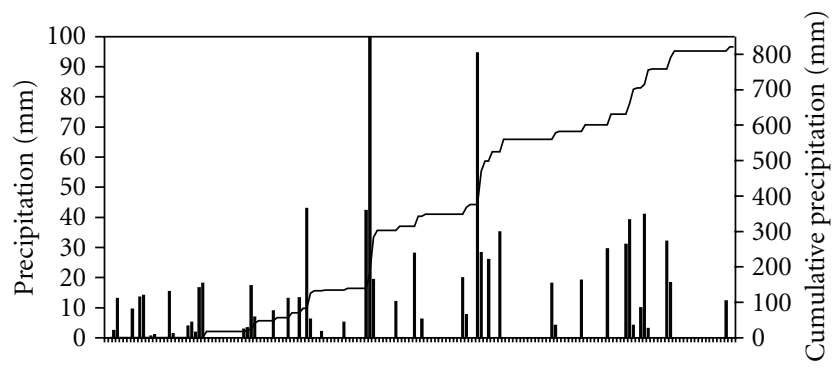

(a) 2008

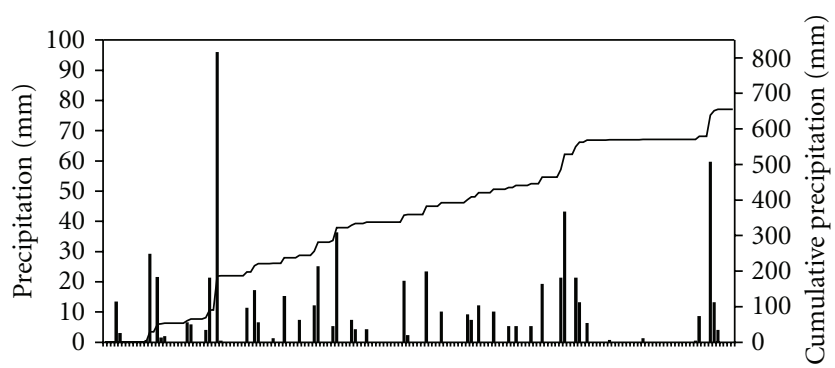

(b) 2009

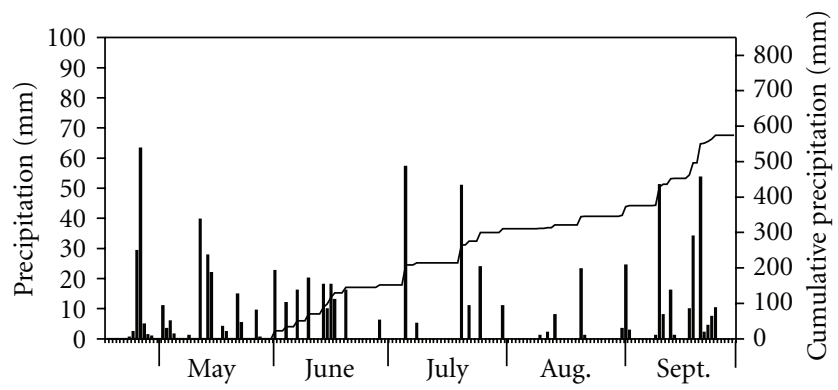

(c) 2010

FIgURE 2: Daily (bars) and cumulative (line) precipitation for nonirrigated treatments in (a) 2008, (b) 2009, and (c) 2010. The solid line starts at the time when corn was planted.

and NDOHIDP treatments was delayed 18 days. The corn hybrids were selected from among the region's high yielding hybrids [31]. In the subsurface-drained and subirrigated treatments, three subsurface drain tile laterals were spaced $6.1 \mathrm{~m}$ apart, and two subsurface drain tile laterals were spaced $12.2 \mathrm{~m}$ apart (Figure 1). They were operated as DNI or DSI. The subirrigation system's water level control devices (AgriDrain, Adair, IA) managed water depth and limited water flow during the winter months as a best management practice to reduce $\mathrm{NO}_{3}-\mathrm{N}$ loss [13].

The site was in a corn-soybean [Glycine max (L.) Merr.] rotation, with corn and soybean present each year (Figure 1). Corn production utilized conventional or reduced tillage in the fall or spring before planting (Table 1), while the soybean crop was no-till planted. Corn was planted (John Deere 7000, Moline, IL) in 75-cm-wide rows at 79,000 seeds ha ${ }^{-1}$ in 2008 and 81,500 seeds ha ${ }^{-1}$ in 2009 and 2010. In 2009, half of the nondrained control was planted on 24 Apr. 2009 and the other half was planted on 12 May due to extremely wet soil conditions in the nondrained control (Figure 1) and nearly complete stand loss in the early planting date. This prevented confounding results due to planting date this year. All plots received broadcast applications of $\mathrm{N}-\mathrm{P}_{2} \mathrm{O}_{5}-\mathrm{K}_{2} \mathrm{O}$ (Table 1) based on University of Missouri soil test recommendations in 2008, but no P and K fertilizer was applied for the 2009 and 2010 growing seasons due to wet soil conditions. Soil test P and $\mathrm{K}$ was high [32] in 2009 and 2010 (data not presented). Corn management practices are summarized in Table 1.

Overhead irrigation application times and amounts were based on the Woodruff scheduling chart [33]. Drainage water management, irrigation dates and amounts are summarized in Table 2. Total cumulative rainfall was $821 \mathrm{~mm}$ in 2008, $655 \mathrm{~mm}$ in 2009, and $574 \mathrm{~mm}$ in 2010 (Figure 2). This exceeded the region's May-September precipitation average of $538 \mathrm{~mm}$ during the past decade [7]. High yielding corn from 1895 to 1998 typically had rainfall of approximately $633 \mathrm{~mm}$ [1]. In this research, supplemental irrigation totaled $108 \mathrm{~mm}$ in 2008, $92 \mathrm{~mm}$ in 2009, and $0 \mathrm{~mm}$ in 2010 (Table 2). Irrigation scheduling was difficult during this "wet" study because overapplication of water could risk fertilizer loss generally as denitrification and root damage on poorly drained claypan soils $[6,8,34]$. That situation may require rescue $\mathrm{N}$ applications [35]. However, anhydrous ammonia was used at a rate that averted the possibility of $\mathrm{N}$ being limiting (Table 1).

Plant height was measured $42 \mathrm{~d}$ after planting in 2009 but not in 2008 and 2010 because treatments showed no differences by visual observation. Plant population was determined before harvest each year by counting a plot length of one harvested row. A plot combine (Kincaid Equipment, Haven, KS in 2008 and Wintersteiger Delta, Salt Lake City, UT in 2009 and 2010) was used to harvest grain and determine its moisture. Grain yield moisture was adjusted to $150 \mathrm{~g} \mathrm{~kg}^{-1}$ before analysis. Grain samples were collected and ten subsamples were analyzed for oil, protein, starch, and extractable starch with a Foss 1241 (Eden Prairie, MN) near infrared spectrometer similar to other research $[5,36]$ using previously established calibrations [37-39]. Grain samples with symptoms of diplodia (Stenocarpella maydis) [40], confirmed with the University of Missouri Plant Diagnostic Clinic, were quantified as the number of grains in the harvested sample of 100 seeds and converted to a percentage of seeds with the disease [36].

The effect of water management system and hybrid was subjected to an ANOVA using PROC GLM [41]. Since planting date was delayed in 2009 and plant populations were reduced over $70 \%$ in the NDNI control compared to DNI or DSI at a $6.1 \mathrm{~m}$ spacing, data for this year were presented separately (Tables 3 and 4). Data from 2008 and 2010 were combined because there was no delay in planting date among treatments and only minimal interactions between years (Tables 5 and 6). Interactions were presented when significant. Main effects were presented in the absence of a significant $(P=0.1$ or 0.05$)$ drainage water management system $\times$ hybrid interaction. Means were separated using Fisher's Protected LSD at $P=0.1$ or 0.05 . 
TABLE 1: Soil organic matter and pH from 0 to $15 \mathrm{~cm}$ and corn management practices in 2008, 2009, and 2010.

\begin{tabular}{|c|c|c|c|}
\hline Soil and site management practices ${ }^{\dagger}$ & 2008 & 2009 & 2010 \\
\hline \multicolumn{4}{|l|}{ Soil test values } \\
\hline Soil organic matter $\left(\mathrm{g} \mathrm{kg}^{-1}\right)$ & $18 \pm 1^{\ddagger}$ & $21 \pm 1$ & $22 \pm 1$ \\
\hline $\mathrm{pH}\left(0.01 \mathrm{M} \mathrm{CaCl}_{2}\right)$ & $6.6 \pm 0.05$ & $6.9 \pm 0.1$ & $6.7 \pm 0.3$ \\
\hline \multicolumn{4}{|l|}{ Fertilizer $\left(\mathrm{N}-\mathrm{P}_{2} \mathrm{O}_{5}-\mathrm{K}_{2} \mathrm{O} \mathrm{kg} \mathrm{ha}{ }^{-1}\right)$} \\
\hline $34-90-180$ & 26 Nov. 2007 & NA & NA \\
\hline $200-0-0$ as anhydrous ammonia & 1 May & 8 Apr. & 27 May \\
\hline \multirow{2}{*}{ Tillage (date) } & Chisel plow (25 Nov. 2007) & & \\
\hline & Tilloll§ (2 May) & Tilloll (23 Apr.) & Tilloll (19 and 20 May) \\
\hline Planting date & 5 May & 24 Apr. & 28 May \\
\hline \multicolumn{2}{|c|}{ Nondrained, nonirrigated delayed planting } & 12 May & \\
\hline \multicolumn{4}{|c|}{ Weed control" } \\
\hline Timing, date & EPOST, 29 May & EPOST, 21 May & PRE, 30 May \\
\hline Herbicide (rate) & $\begin{array}{l}\text { Glyphosate } \\
\left(0.84 \mathrm{~kg}^{-1} \mathrm{ha}^{-1}\right)+\text { DAS } \\
\left(20 \mathrm{~g} \mathrm{~L}^{-1}\right)+\text { premixture of } \\
\text { atrazine }+ \text { S-metolachlor }+ \\
\text { mesotrione }\left(3.3 \mathrm{~kg} \mathrm{ai} \mathrm{ha}^{-1}\right)\end{array}$ & $\begin{array}{l}\text { glyphosate }\left(0.84 \mathrm{~kg} \text { ae } \mathrm{ha}^{-1}\right)+ \\
\text { premixture of atrazine }+ \\
\text { dimethenamid-P } \\
\left(3.5 \mathrm{~kg}^{-1} \mathrm{ha}^{-1}\right)\end{array}$ & $\begin{array}{l}\text { Saflufenacil }\left(0.025 \mathrm{~kg} \mathrm{ai} \mathrm{ha}^{-1}\right)+ \\
\text { glyphosate }\left(0.84 \mathrm{~kg} \mathrm{ae} \mathrm{ha}^{-1}\right)\end{array}$ \\
\hline Timing, date & & POST, 25 June & POST, 22 June \\
\hline Herbicide (rate) & & $\begin{array}{l}\text { Glyphosate }\left(1.2 \mathrm{~kg} \mathrm{ae} \mathrm{ha}^{-1}\right)+ \\
\text { DAS }\left(20 \mathrm{~g} \mathrm{~L}^{-1}\right)\end{array}$ & $\begin{array}{l}\text { Premixture of acetochlor }+ \\
\text { atrazine }\left(5 \mathrm{~kg} \mathrm{ai} \mathrm{ha}^{-1}\right)\end{array}$ \\
\hline Timing, date & & & LPOST, 29 June \\
\hline Herbicide (rate) & & & $\begin{array}{l}\text { Glyphosate }\left(0.84 \mathrm{~kg} \mathrm{ae} \mathrm{ha}^{-1}\right)+ \\
\text { DAS }\left(20 \mathrm{~g} \mathrm{~L}^{-1}\right)\end{array}$ \\
\hline \multicolumn{4}{|c|}{$\begin{array}{l}{ }^{\dagger} \text { Abbreviations: EPOST: early postemergence; DAS: diammonium sulfate; LPOST: late postemergence; NA: none applied; PRE: preemergence. } \\
{ }^{\ddagger} \text { Standard deviation. } \\
\text { \$ Tilloll 875, Marysville, KS. } \\
{ }^{9} \text { Herbicide chemical names: acetochlor: (2-chloro-2'-methyl-6'-ethyl-N-ethoxymethylacetanilide); atrazine: (6-chloro-N-ethyl-N'-(1-methylethyl)- } \\
\text { 1,3,5-triazine-2,4-diamine); dimethenamid-P: (2-chloro-N-[(1-methyl-2-methoxy)ethyl]-N-(2,4-dimethyl-thien-3-yl)-acetamide); glyphosate: (N-(phos- } \\
\text { phonomethyl)glycine); mesotrione: (2-[4-(methylsulfonyl)-2-nitrobenzoyl]-1,3-cyclohexanedione); S-metolachlor: (2-chloro-N-(2-ethyl-6-methylphen- } \\
\text { yl)-N-(2-methoxy-1-methylethyl)acetamide); saflufenacil: ( } \mathrm{N}^{\prime} \text {-[2-chloro-4-fluoro-5-(3-methyl-2,6-dioxo-4-(trifluoromethyl)-3,6-dihydro-1(2H)-pyrimid- } \\
\text { inyl)benzoyl]-N-isopropyl-N-methylsulfamide). }\end{array}$} \\
\hline
\end{tabular}

TABLE 2: Drainage and irrigation water management schedule by year.

\begin{tabular}{|c|c|c|c|}
\hline Water management schedule & 2008 & 2009 & 2010 \\
\hline Drainage mode & 15 Mar. & 15 Mar. & 15 Mar. \\
\hline Controlled drainage date & 17 July & 25 June & 6 July \\
\hline Subirrigation date & 17 July-25 July, 4 Aug.-15 Sep. & 25 June-16 Sep. & 2 Aug.-10 Sep. \\
\hline Drainage mode & 25 July-4 Aug., 15 Sep. & 16 Sep. & 10 Sep. -29 Oct. \\
\hline Controlled drainage & 15 Nov. & 22 Nov. & 29 Oct. \\
\hline \multirow{4}{*}{ Overhead irrigation dates and amounts } & 18 July, $13 \mathrm{~mm}$ & 1 July, 34 mm & None applied \\
\hline & 8 Aug., 43 mm & 20 July, $21 \mathrm{~mm}$ & \\
\hline & 19 Aug., $32 \mathrm{~mm}$ & 30 July, 25 mm & \\
\hline & 28 Aug., $20 \mathrm{~mm}$ & 6 Aug., $12 \mathrm{~mm}$ & \\
\hline
\end{tabular}

TABLE 3: ANOVA table of water management (WM) and corn hybrid treatments for height, plant population, grain moisture, yield, and diplodia in 2009.

\begin{tabular}{lccccccccccc}
\hline \multirow{2}{*}{ Source } & \multicolumn{4}{c}{ Height } & \multicolumn{2}{c}{ Population } & \multicolumn{2}{c}{ Moisture } & \multicolumn{2}{c}{ Yield } & \multicolumn{2}{c}{ Diplodia } \\
& $\mathrm{df}$ & $F$-value & $\operatorname{Pr}>F$ & $F$-value & $\operatorname{Pr}>F$ & $F$-value & $\operatorname{Pr}>F$ & $F$-value & $\operatorname{Pr}>F$ & $F$-value & $\operatorname{Pr}>F$ \\
\hline Rep & 3 & 2.9 & 0.035 & 0.5 & 0.687 & 1.7 & 0.162 & 0.6 & 0.646 & 24.0 & $<0.0001$ \\
WM & 7 & 13.1 & $<0.0001$ & 27.4 & $<0.0001$ & 3.1 & 0.016 & 12.0 & $<0.0001$ & 4.0 & 0.004 \\
Rep $\times$ WM & 21 & 2.9 & $<0.0001$ & 3.1 & $<0.0001$ & 1.8 & 0.025 & 1.4 & 0.138 & 5.5 & $<0.0001$ \\
Hybrid & 4 & 0.6 & 0.669 & 0.6 & 0.644 & 0.3 & 0.909 & 1.3 & 0.275 & 13.3 & $<0.0001$ \\
WM $\times$ hybrid & 28 & 1.7 & 0.012 & 1.0 & 0.454 & 1.6 & 0.028 & 1.2 & 0.205 & 0.9 & 0.650 \\
\hline
\end{tabular}


TABLE 4: ANOVA table of water management (WM) and corn hybrid treatments for grain oil, protein, starch, and extractable starch concentration in 2009.

\begin{tabular}{|c|c|c|c|c|c|c|c|c|c|}
\hline \multirow{2}{*}{ Source } & \multirow[b]{2}{*}{$\mathrm{df}$} & \multicolumn{2}{|c|}{ Oil } & \multicolumn{2}{|c|}{ Protein } & \multicolumn{2}{|c|}{ Starch } & \multicolumn{2}{|c|}{ Extractable starch } \\
\hline & & $F$-value & $\operatorname{Pr}>F$ & $F$-value & $\operatorname{Pr}>F$ & $F$-value & $\operatorname{Pr}>F$ & $F$-value & $\operatorname{Pr}>F$ \\
\hline Rep & 3 & 4.3 & 0.006 & 7.2 & 0.0001 & 0.5 & 0.678 & 14.6 & $<0.0001$ \\
\hline WM & 7 & 5.6 & 0.0004 & 14.6 & $<0.0001$ & 1.4 & 0.238 & 6.6 & 0.0001 \\
\hline $\operatorname{Rep} \times W M$ & 21 & 2.3 & 0.001 & 6.9 & $<0.0001$ & 0.7 & 0.850 & 3.8 & $<0.0001$ \\
\hline Hybrid & 4 & 6.9 & $<0.0001$ & 13.0 & $<0.0001$ & 0.2 & 0.938 & 22.6 & $<0.0001$ \\
\hline $\mathrm{WM} \times$ hybrid & 28 & 1.0 & 0.533 & 1.0 & 0.457 & 0.6 & 0.962 & 1.3 & 0.183 \\
\hline
\end{tabular}

TABLE 5: ANOVA table of water management (WM) and corn hybrid treatments for plant population, grain moisture, yield, and diplodia in 2008 and 2010.

\begin{tabular}{|c|c|c|c|c|c|c|c|c|c|}
\hline \multirow{2}{*}{ Source } & \multirow[b]{2}{*}{$\mathrm{df}$} & \multicolumn{2}{|c|}{ Population } & \multicolumn{2}{|c|}{ Moisture } & \multicolumn{2}{|c|}{ Yield } & \multicolumn{2}{|c|}{ Diplodia } \\
\hline & & $F$-value & $\operatorname{Pr}>F$ & $F$-value & $\operatorname{Pr}>F$ & $F$-value & $\operatorname{Pr}>F$ & $F$-value & $\operatorname{Pr}>F$ \\
\hline Year & 1 & 94.4 & $<0.0001$ & 578.4 & $<0.0001$ & 0.8 & 0.38 & 151.1 & $<0.0001$ \\
\hline Year $\times$ rep & 6 & 1.0 & 0.449 & 0.5 & 0.801 & 2.6 & 0.049 & 0.8 & 0.544 \\
\hline WM & 5 & 0.8 & 0.571 & 3.9 & 0.008 & 1.9 & 0.116 & 1.0 & 0.463 \\
\hline Year $\times W M$ & 5 & 5.3 & $<0.0001$ & 15.7 & $<0.0001$ & 15.8 & $<0.0001$ & 6.9 & $<0.0001$ \\
\hline Year $\times$ rep $\times$ WM & 30 & 6.7 & $<0.0001$ & 4.1 & $<0.0001$ & 8.1 & $<0.0001$ & 7.2 & $<0.0001$ \\
\hline Hybrid & 4 & 3.8 & 0.005 & 205.4 & $<0.0001$ & 11.8 & $<0.0001$ & 15.2 & $<0.0001$ \\
\hline Year $\times$ hybrid & 4 & 4.0 & 0.003 & 7.3 & $<0.0001$ & 2.1 & 0.081 & 13.0 & $<0.0001$ \\
\hline WM $\times$ hybrid & 20 & 1.3 & 0.159 & 1.5 & 0.094 & 2.2 & 0.001 & 1.2 & 0.277 \\
\hline Year $\times \mathrm{WM} \times$ hybrid & 20 & 0.8 & 0.668 & 0.5 & 0.965 & 1.1 & 0.382 & 1.1 & 0.304 \\
\hline
\end{tabular}

TABLE 6: ANOVA table of water management (WM) and corn hybrid treatments for grain oil, protein, starch, and extractable starch concentration in 2008 and 2010.

\begin{tabular}{|c|c|c|c|c|c|c|c|c|c|}
\hline \multirow{2}{*}{ Source } & \multirow[b]{2}{*}{ df } & \multicolumn{2}{|c|}{ Oil } & \multicolumn{2}{|c|}{ Protein } & \multicolumn{2}{|c|}{ Starch } & \multicolumn{2}{|c|}{ Extractable starch } \\
\hline & & $F$-value & $\operatorname{Pr}>F$ & $F$-value & $\operatorname{Pr}>F$ & $F$-value & $\operatorname{Pr}>F$ & $F$-value & $\operatorname{Pr}>F$ \\
\hline Year & 1 & 17.3 & 0.0002 & 0.1 & 0.776 & 76.1 & $<0.0001$ & 14.5 & 0.0006 \\
\hline Year $\times$ rep & 6 & 0.55 & 0.737 & 1.5 & 0.235 & 1.3 & 0.310 & 0.7 & 0.631 \\
\hline WM & 5 & 0.83 & 0.540 & 2.0 & 0.111 & 2.2 & 0.079 & 1.8 & 0.144 \\
\hline Year $\times W M$ & 5 & 1.2 & 0.307 & 11.5 & $<0.0001$ & 7.9 & $<0.0001$ & 8.8 & $<0.0001$ \\
\hline Year $\times$ rep $\times W M$ & 30 & 1.5 & 0.058 & 5.8 & $<0.0001$ & 3.6 & $<0.0001$ & 4.9 & $<0.0001$ \\
\hline Hybrid & 4 & 142.2 & $<0.0001$ & 32.3 & $<0.0001$ & 21.8 & $<0.0001$ & 62.6 & $<0.0001$ \\
\hline Year $\times$ hybrid & 4 & 18.6 & $<0.0001$ & 1.4 & 0.226 & 9.6 & $<0.0001$ & 10.2 & $<0.0001$ \\
\hline WM $\times$ hybrid & 20 & 1.9 & 0.011 & 1.1 & 0.306 & 1.5 & 0.089 & 1.1 & 0.382 \\
\hline Year $\times \mathrm{WM} \times$ hybrid & 20 & 0.7 & 0.791 & 0.8 & 0.774 & 1.0 & 0.422 & 0.5 & 0.951 \\
\hline
\end{tabular}

TABLE 7: Effects of water management and hybrid interaction on plant heights $42 \mathrm{~d}$ after 24 April planting date in 2009.

\begin{tabular}{lcccc}
\hline Water management system & DKC63-42 & LG2642 & $\begin{array}{c}\text { Asgrow785 } \\
\text { cm }\end{array}$ & Kruger2114 DKC61-73 \\
\hline Nondrained, nonirrigated (NDNI) & 28 & 36 & 28 & 67 \\
Nondrained, nonirrigated, delayed planting (NDNIDP) & 41 & 53 & 46 & 53 \\
Drained, nonirrigated (DNI) at 6.1 m & 82 & 77 & 73 & 73 \\
Drained, nonirrigated (DNI) at 12.2 m & 59 & 65 & 61 & 54 \\
Drained plus subirrigated (DSI) at 6.1 m & 77 & 76 & 60 & 72 \\
Drained plus subirrigated (DSI) at 12.2 m & 53 & 60 & 49 & 58 \\
Overhead irrigated, nondrained (NDOHI) & 49 & 24 & 45 & 78 \\
Overhead irrigated, nondrained, delayed planting (NDOHIDP) & 43 & 49 & 50 & 48 \\
LSD $(P=0.05)$ & & & 30 & 46 \\
\hline
\end{tabular}


TABLE 8: Water management main effects for plant population before harvest and grain yield, moisture, diplodia, oil, protein, starch, and extractable starch in 2009.

\begin{tabular}{|c|c|c|c|c|c|c|c|c|}
\hline Water management system & $\begin{array}{c}\text { Plant } \\
\text { population } \\
\text { No. } \text { ha }^{-1}\end{array}$ & $\begin{array}{l}\text { Yield } \\
\mathrm{Mg} \mathrm{ha}^{-1}\end{array}$ & $\begin{array}{c}\text { Moisture } \\
\mathrm{g} \mathrm{kg}^{-1}\end{array}$ & $\begin{array}{r}\text { Diplodia } \\
\%\end{array}$ & $\begin{array}{l}\text { Oil } \\
\mathrm{g} \mathrm{kg}^{-1}\end{array}$ & $\begin{array}{l}\text { Protein } \\
\mathrm{g} \mathrm{kg}^{-1}\end{array}$ & $\begin{array}{l}\text { Starch } \\
\mathrm{g} \mathrm{kg}^{-1}\end{array}$ & $\begin{array}{l}\text { Extractable } \\
\text { starch } \\
\qquad \mathrm{g} \mathrm{kg}^{-1}\end{array}$ \\
\hline Nondrained, nonirrigated (NDNI) & 13,600 & 4.5 & 14.5 & 21 & 41.3 & 95.2 & 711 & 657 \\
\hline $\begin{array}{l}\text { Nondrained, nonirrigated, delayed } \\
\text { planting (NDNIDP) }\end{array}$ & 55,600 & 14.4 & 19.3 & 8 & 43.2 & 89.4 & 709 & 662 \\
\hline Drained, nonirrigated (DNI) at $6.1 \mathrm{~m}$ & 47,400 & 9.2 & 17.1 & 23 & 39.8 & 94.4 & 714 & 660 \\
\hline Drained, nonirrigated (DNI) at $12.2 \mathrm{~m}$ & 33,800 & 7.6 & 15.3 & 21 & 39.8 & 95.0 & 702 & 659 \\
\hline Drained plus subirrigated (DSI) at $6.1 \mathrm{~m}$ & 45,200 & 9.8 & 17.6 & 18 & 39.8 & 92.9 & 713 & 661 \\
\hline Drained plus subirrigated (DSI) at $12.2 \mathrm{~m}$ & 25,900 & 6.7 & 14.6 & 20 & 40.0 & 95.9 & 712 & 657 \\
\hline $\begin{array}{l}\text { Overhead irrigated, nondrained } \\
\text { (NDOHI) }\end{array}$ & 6,700 & 2.6 & 13.5 & 15 & 41.9 & 85.2 & 716 & 673 \\
\hline $\begin{array}{l}\text { Overhead irrigated, nondrained, delayed } \\
\text { planting (NDOHIDP) }\end{array}$ & 54,300 & 12.8 & 19.4 & 9 & 40.6 & 86.4 & 714 & 673 \\
\hline $\operatorname{LSD}(P=0.05)$ & 17,800 & 3.0 & 3.9 & 9 & 2.2 & 7.7 & NS & 11 \\
\hline
\end{tabular}

TABLE 9: Hybrid main effects for grain diplodia, oil, protein, and extractable starch in 2009.

\begin{tabular}{lcccc}
\hline Hybrid & $\begin{array}{c}\text { Diplodia } \\
\%\end{array}$ & $\begin{array}{c}\text { Oil } \\
\mathrm{g} \mathrm{kg}^{-1}\end{array}$ & $\begin{array}{c}\text { Protein } \\
\mathrm{g} \mathrm{kg}^{-1}\end{array}$ & $\begin{array}{c}\text { Extractable starch } \\
\mathrm{g} \mathrm{kg}^{-1}\end{array}$ \\
\hline DKC63-42 & 23 & 40.9 & 94.3 & 660 \\
LG2642 & 8 & 41.7 & 89.6 & 668 \\
Asgrow785 & 15 & 39.6 & 90.7 & 669 \\
Kruger2114 & 33 & 39.3 & 98.4 & 645 \\
DKC61-73 & 17 & 39.7 & 95.3 & 661 \\
\hline LSD $(P=0.05)$ & 5 & 0.9 & 1.8 & 3 \\
\hline
\end{tabular}

\section{Results and Discussion}

3.1. Delayed Planting (2009). Corn plant height $42 \mathrm{~d}$ after planting was affected $(P=0.012)$ by drainage water management and hybrid selection (Table 7). In general, the tallest plants were in the DNI and DSI at $6.1 \mathrm{~m}$ spacing. However, corn height for drainage or DSI 6 was similar to DSI 12, except for Asgrow785 planted into DSI 12. Although the NDNIDP was planted $18 \mathrm{~d}$ later, heights were similar to the early planted nondrained corn. These data indicated better growing conditions for plants in the DNI and DSI treatments compared to their NDNI and NDOHI counterparts. This was evident in final plant population at harvest (Table 8). Nearly all plants in the NDNI or NDOHI controls were eliminated by early wet conditions. DSI 6 had $74 \%$ greater plant population than DSI 12 , due to the closer spacing's more efficient drainage. Typically, a delay in planting date results in a lower yield, but in a wet year it was beneficial to wait until the seedbed dried adequately to establish plants well.

Grain yield was ranked NDNIDP $=$ NDOHIDP > DSI $6=$ DNI $6=$ DNI $12 \geq$ DSI $12 \geq$ NDNI $=$ NDOHI. Differences in drain tile spacing were probably due to lateral flow limitations caused by the claypan [23]. DNI 6, DSI 6 , and DNI 12 increased yield from 4.7 to $7.2 \mathrm{Mgha}^{-1}$ compared to the nondrained (NDNI and NDOHI) controls.
Subsurface drain tiles in a claypan soil in central Missouri at a $15.3 \mathrm{~m}$ spacing removed from $41 \%$ to $47 \%$ of excess precipitation [23]. Overhead irrigation or subirrigation appeared to decrease yield, though it was not significantly different than appropriate nonirrigated controls in 2009 with its high rainfall that made scheduling difficult. This scenario has been common in other water management research on claypan soils $[6,8,23]$. Sustained rainfall throughout the summer months allowed the DNIDP and NDOHIDP controls to produce high yields $\left(>12 \mathrm{Mgha}^{-1}\right)$, which is atypical for these soils. In general, grain moisture was higher and diplodia was lower with late-planted NDNIDP and NDOHIDP corn.

Oil and protein concentrations usually are inversely related [39]. The NDNIDP had the highest oil $\left(43.2 \mathrm{~g} \mathrm{~kg}^{-1}\right)$ concentration, and delayed planting controls had the lowest protein ( 85.2 to $86.4 \mathrm{~g} \mathrm{~kg}^{-1}$ ) concentration (Table 8 ). Water management did not affect starch concentration, but extractable starch was highest $\left(673 \mathrm{~g} \mathrm{~kg}^{-1}\right)$ in the overheadirrigated controls. The effect of water management on grain quality was due primarily to planting date and differences in population at harvest. In a year with consistent summer rainfall, stand establishment was essential for high yielding corn.

Since no interaction between drainage water management and high yielding hybrid was detected in 2009 (Tables 3 and 4), main effects for hybrid are presented in Table 9. 
TABle 10: Corn grain yield, moisture, oil, and protein response to water management systems and hybrid in 2008 and 2010 . Data were combined over years.

\begin{tabular}{|c|c|c|c|c|c|}
\hline Water management system ${ }^{\dagger}$ & Hybrid & $\begin{array}{c}\text { Yield } \\
\text { Mg ha }^{-1}\end{array}$ & $\begin{array}{c}\text { Moisture } \\
\mathrm{g} \mathrm{kg}^{-1}\end{array}$ & $\begin{array}{c}\text { Oil } \\
\mathrm{g} \mathrm{kg}^{-1}\end{array}$ & $\begin{array}{l}\text { Starch } \\
\mathrm{g} \mathrm{kg}^{-1}\end{array}$ \\
\hline \multirow{5}{*}{ NDNI } & DKC63-42 & 11.1 & 167 & 41.3 & 725 \\
\hline & LG2642 & 10.8 & 179 & 43.8 & 721 \\
\hline & Asgrow785 & 10.5 & 158 & 39.5 & 727 \\
\hline & Kruger2114 & 10.3 & 175 & 41.6 & 733 \\
\hline & DKC61-73 & 9.9 & 155 & 39.0 & 725 \\
\hline \multirow{5}{*}{ DNI 6} & DKC63-42 & 12.2 & 160 & 41.7 & 724 \\
\hline & LG2642 & 11.7 & 178 & 43.4 & 720 \\
\hline & Asgrow785 & 12.1 & 155 & 37.7 & 729 \\
\hline & Kruger2114 & 11.2 & 174 & 41.9 & 723 \\
\hline & DKC61-73 & 12.1 & 151 & 39.3 & 722 \\
\hline \multirow{5}{*}{ DNI 12} & DKC63-42 & 11.7 & 164 & 42.0 & 725 \\
\hline & LG2642 & 11.8 & 181 & 43.8 & 720 \\
\hline & Asgrow785 & 11.6 & 154 & 38.7 & 727 \\
\hline & Kruger2114 & 11.2 & 179 & 41.6 & 723 \\
\hline & DKC61-73 & 11.2 & 152 & 38.3 & 725 \\
\hline \multirow{5}{*}{ DSI 6} & DKC63-42 & 11.6 & 166 & 41.9 & 724 \\
\hline & LG2642 & 11.1 & 175 & 43.2 & 722 \\
\hline & Asgrow785 & 11.0 & 157 & 40.2 & 726 \\
\hline & Kruger2114 & 10.5 & 174 & 40.8 & 728 \\
\hline & DKC61-73 & 10.8 & 149 & 38.2 & 727 \\
\hline \multirow{5}{*}{ DSI 12} & DKC63-42 & 12.1 & 168 & 41.5 & 726 \\
\hline & LG2642 & 11.1 & 177 & 43.5 & 722 \\
\hline & Asgrow785 & 10.7 & 153 & 38.3 & 730 \\
\hline & Kruger2114 & 11.1 & 177 & 41.6 & 726 \\
\hline & DKC61-73 & 10.4 & 151 & 37.9 & 727 \\
\hline \multirow{5}{*}{ NDOHI } & DKC63-42 & 10.7 & 163 & 42.7 & 724 \\
\hline & LG2642 & 11.4 & 175 & 43.5 & 723 \\
\hline & Asgrow785 & 11.0 & 156 & 38.3 & 729 \\
\hline & Kruger2114 & 10.6 & 172 & 42.7 & 724 \\
\hline & DKC61-73 & 10.2 & 153 & 37.3 & 728 \\
\hline LSD & & $1.1^{\ddagger}$ & $8^{\S}$ & $2.1^{\ddagger}$ & $5^{\S}$ \\
\hline
\end{tabular}

Plant populations were low $\left(<38,000\right.$ plants ha $\left.^{-1}\right)$, but no differences were found in plant population, moisture, yield, and starch concentration among hybrids (Tables 3 and 4). Grain with diplodia, which was due to hybrid differences, ranged from $8 \%$ for LG2642 to 33\% for Kruger2114. Oil concentration was greatest with LG2642 $\left(41.7 \mathrm{~g} \mathrm{~kg}^{-1}\right)$ and DKC63-42 (40.9 $\left.\mathrm{g} \mathrm{kg}^{-1}\right)$. Protein concentration $98.4 \mathrm{~g} \mathrm{~kg}^{-1}$ with Kruger2114 and $89.6 \mathrm{~g} \mathrm{~kg}^{-1}$ with LG2642.

3.2. No Delayed Planting (2008 and 2010). Data were pooled over 2008 and 2010 since no significant three-way interactions (year $\times$ water management $\times$ hybrid) were detected for dependent variables (Tables 5 and 6). In 2008 and 2010, grain yield was 9.9 to $11.1 \mathrm{Mgha}^{-1}$ in the NDNI control with DKC63-42 yielding 1.2 $\mathrm{Mg} \mathrm{ha}^{-1}$ greater than DKC61-73 (Table 10). When compared to the NDNI control, DNI at $6.1 \mathrm{~m}$ increased yield of all hybrids from 1.1 to $2.2 \mathrm{Mg} \mathrm{ha}^{-1}$. At a $12.2 \mathrm{~m}$ spacing, grain yield increased with Asgrow785 and DKC61-73 1.1 and 1.3 $\mathrm{Mg} \mathrm{ha}^{-1}$, respectively. This indicates either that DKC61-73 was more susceptible to water stress or that with appropriate drainage it could reach its production potential more so than other hybrids. None of the DSI treatments affected yield compared to the NDNI except DKC61-73 at $6.1 \mathrm{~m}$, indicating that the subirrigation should have remained in drainage mode throughout the season for optimal crop production. Similarly, overhead irrigation had no effect on yield in extremely wet years similar to 2009 .

Grain moisture with Asgrow 785 and DKC61-73 was generally drier than the other hybrids regardless of the drainage 
TABLE 11: Hybrid main effects for plant population before harvest, diplodia, protein, and extractable starch in 2008 and 2010 . Data were combined over years in the absence of an interaction.

\begin{tabular}{|c|c|c|c|c|c|c|}
\hline \multirow{3}{*}{ Hybrid } & \multicolumn{2}{|c|}{ Plant population } & \multicolumn{2}{|c|}{ Diplodia } & \multirow{3}{*}{$\begin{array}{l}\text { Protein } \\
\mathrm{g} \mathrm{kg}^{-1}\end{array}$} & \multirow{3}{*}{$\begin{array}{c}\text { Extractable starch } \\
\qquad \mathrm{g} \mathrm{kg}^{-1}\end{array}$} \\
\hline & 2008 & 2010 & 2008 & 2010 & & \\
\hline & \multicolumn{2}{|c|}{ no. $\mathrm{ha}^{-1}$} & \multicolumn{2}{|c|}{$\%$} & & \\
\hline DKC63-42 & 71,600 & 63,000 & 15 & 3 & 78.0 & 686 \\
\hline LG2642 & 71,900 & 60,300 & 13 & 1 & 74.8 & 686 \\
\hline Asgrow785 & 71,100 & 60,500 & 12 & 3 & 75.1 & 693 \\
\hline Kruger2114 & 71,900 & 58,000 & 14 & 1 & 78.7 & 673 \\
\hline DKC61-73 & 70,900 & 59,500 & 19 & 3 & 81.4 & 686 \\
\hline $\operatorname{LSD}(P=0.05)$ & NS & 2,200 & 2 & 1 & 1.2 & 2 \\
\hline
\end{tabular}

system (Table 10). DSI 12 increased grain moisture by $8 \mathrm{~g} \mathrm{~kg}^{-1}$ compared to DNI 6. The greatest disparity in grain moisture among hybrids was between DNI 6 and DNI 12 compared to the other water management systems. Although a significant interaction emerged between drainage water management and hybrid for oil and starch concentration (Table 6), there were no consistent effects of drainage water management on oil and starch concentrations (Table 10). This was consistent with research evaluating soybean grain quality response to drainage water management [27, 29], while Wiersma et al. [42] showed a $6 \mathrm{~g} \mathrm{~kg}^{-1}$ increase in protein concentration as the drainage coefficient increased to $2 \mathrm{~cm} \mathrm{day}^{-1}$. There was no effect of drainage water management on harvested plant population (from 63,000 to 67,700 plants $\left.\mathrm{ha}^{-1}\right)$, diplodia $(8-10 \%)$, protein $(76.1-$ $\left.80.2 \mathrm{~g} \mathrm{~kg}^{-1}\right)$, or extractable starch $\left(682-687 \mathrm{~g} \mathrm{~kg}^{-1}\right)$ concentration, although differences between years were detected (Table 6).

Plant population at harvest was similar among hybrids in 2008. However, DKC63-42 had 2,500-5,000 plants ha ${ }^{-1}$ greater than the other hybrids (Table 11), which could have contributed toward higher overall yields in the NDNI, DNI 6, DSI 6, and DSI 12 treatments compared to the other hybrids (Table 10). Grain with diplodia symptoms was inconsistent between hybrids in 2008 and 2010 (Table 11). Diplodia was greatest in both years with DKC63-42 and DKC6173 and lowest in both years with LG2642 and Kruger2114. Protein concentration in those years was from 74.8 to $81.4 \mathrm{~g} \mathrm{~kg}^{-1}$, which was from 13.9 to $19.7 \mathrm{~g} \mathrm{~kg}^{-1}$ less than 2009. Extractable starch was greatest with Asgrow785 and lowest with Kruger2114. Similarly, differences in soybean oil concentration depended more on the soybean cultivar than on water management [27].

\section{Conclusion}

In three wet years, DNI 6 increased yield from 1.1 to $6.6 \mathrm{Mgha}^{-1}$ compared to the nondrained (NDNI or NDOHI) controls. In 2009, the NDNIDP control yields were from 4.6 to $5.2 \mathrm{Mg} \mathrm{ha}^{-1}$ greater than DNI or DSI, due to an even distribution of summer rains. Grain yields were similar in the 6.1- and $12.2 \mathrm{~m}$-spaced DNI and DSI systems in 2008 and 2010, but plant population increased 74\% and yields were $3.1 \mathrm{Mg} \mathrm{ha}^{-1}$ greater with DSI 6 compared to DSI
12 in 2009. In this study, drainage helped hybrids increase yields from $10 \%$ to over $50 \%$ in years with high rainfall. Differences among hybrids may be due to their tolerance of saturated conditions. Corn hybrids achieved their yield potential when adequate drainage was provided, and some hybrids had greater wetness tolerance compared to others. Some high yielding hybrids tested in this study reached a higher yield potential using drainage water management systems versus nondrained soil. Drainage is an important component of high yielding corn production systems.

\section{Nomenclature}

Corn: Zea mays L. “Asgrow785," “DKC61-73,” "DKC63-42," “LG2642,” "Kruger2114”.

\section{Abbreviations}

DNI 6: $\quad$ Drained, nonirrigated (6.1 $\mathrm{m}$ drain spacing)

DNI 12: $\quad$ Drained, nonirrigated (12.2 $\mathrm{m}$ drain spacing)

DSI 6: $\quad$ Drained, subirrigated (6.1 $\mathrm{m}$ drain spacing)

DSI 12: $\quad$ Drained, subirrigated (12.2 $\mathrm{m}$ drain spacing)

NDNI: $\quad$ Nondrained, nonirrigated

NDNIDP: Nondrained, nonirrigated, delayed planting

NDOHI: Nondrained, overhead irrigated

NDOHIDP: Overhead irrigated, nondrained, delayed planting.

\section{Acknowledgments}

The authors would like to thank Katherine Ausmus, Kolton Bevill, Brett Craigmyle, Chris Dudenhoeffer, Miranda Glasgow, Hayden Hunziker, Clinton Meinhardt, and John Shetley for their technical assistance.

\section{References}

[1] Q. Hu and G. Buyanovsky, "Climate effects on corn yield in Missouri," Journal of Applied Meteorology, vol. 42, no. 11, pp. 1626-1635, 2003.

[2] C. J. Kucharik, "A multidecadal trend of earlier corn planting in the central USA," Agronomy Journal, vol. 98, no. 6, pp. 15441550, 2006. 
[3] J. G. Lauer, P. R. Carter, T. M. Wood et al., "Corn hybrid response to planting date in the northern corn belt," Agronomy Journal, vol. 91, no. 5, pp. 834-839, 1999.

[4] P. Jiang, N. R. Kitchen, S. H. Anderson, E. J. Sadler, and K. A. Sudduth, "Estimating plant-available water using the simple inverse yield model for claypan landscapes," Agronomy Journal, vol. 100, no. 3, pp. 830-836, 2008.

[5] K. A. Nelson, P. P. Motavalli, R. L. Smoot et al., "Utility of dried distillers grain as a fertilizer source for corn," Journal of Agricultural Science, vol. 1, no. 1, pp. 3-12, 2009.

[6] K. A. Nelson, S. M. Paniagua, and P. P. Motavalli, "Effect of polymer coated urea, irrigation, and drainage on nitrogen utilization and yield of corn in a claypan soil," Agronomy Journal, vol. 101, no. 3, pp. 681-687, 2009.

[7] K. A. Nelson, R. L. Smoot, and B. A. Burdick, "Seed coat technology affects yields of relay intercrop, full season, and double crop soybean in upstate Missouri," Crop Management. In press.

[8] A. L. Thompson, C. J. Gantzer, and S. H. Anderson, "Topsoil depth, fertility, water management, and weather influences on yield," Soil Science Society of America Journal, vol. 55, no. 4, pp. 1085-1091, 1991.

[9] D. W. Blevins, D. H. Wilkison, B. P. Kelly, and S. R. Silva, "Movement of nitrate fertilizer to glacial till and runoff from a claypan soil," Journal of Environmental Quality, vol. 25, no. 3, pp. 584-593, 1996.

[10] H. Blanco-Canqui, C. J. Gantzer, S. H. Anderson, E. E. Alberts, and F. Ghidey, "Saturated hydraulic conductivity and its impact on simulated runoff for claypan soils," Soil Science Society of America Journal, vol. 66, no. 5, pp. 1596-1602, 2002.

[11] S. T. Chieng, J. Keng, and M. G. Driehuyzen, "Effects of subsurface drainage and subirrigation on the yields of four crops," Canadian Agricultural Engineering, vol. 29, no. 1, pp. 21-26, 1987.

[12] C. F. Drury, C. S. Tan, J. D. Gaynor, T. O. Oloya, I. J. Van Wesenbeeck, and D. J. McKenney, "Optimizing corn production and reducing nitrate losses with water table controlsubirrigation," Soil Science Society of America Journal, vol. 61, no. 3, pp. 889-895, 1997.

[13] J. Frankenberger, E. Kladivko, G. Sands, D. Jaynes, N. Fausey, M. Helmers et al., "Drainage water management for the Midwest: questions and answers about drainage water management for the Midwest," Purdue Extension, pp. 8, 2006.

[14] D. L. Thomas, P. G. Hunt, and J. W. Gilliam, "Water table management for water quality improvement," Journal of Soil \& Water Conservation, vol. 47, no. 1, pp. 65-70, 1992.

[15] C. F. Drury and C. S. Tan, "Long-term (35 years) effects of fertilization, rotation and weather on corn yields," Canadian Journal of Plant Science, vol. 75, no. 2, pp. 355-362, 1995.

[16] M. J. Fisher, N. R. Fausey, S. E. Subler, L. C. Brown, and P. M. Bierman, "Water table management, nitrogen dynamics, and yields of corn and soybean," Soil Science Society of America Journal, vol. 63, no. 6, pp. 1786-1795, 1999.

[17] E. J. Kladivko, J. Grochulska, R. F. Turco, G. E. Van Scoyoc, and J. D. Eigel, "Pesticide and nitrate transport into subsurface tile drains of different spacings," Journal of Environmental Quality, vol. 28, no. 3, pp. 997-1004, 1999.

[18] N. R. Fausey, L. C. Brown, H. W. Belcher, and R. S. Kanwar, "Drainage and water quality in Great Lakes and cornbelt states," Journal of Irrigation \& drainage Engineering, vol. 121, no. 4, pp. 283-288, 1995.
[19] C. F. Drury, C. S. Tan, J. D. Gaynor, T. O. Oloya, and T. W. Welacky, "Water quality: influence of controlled drainagesubirrigation on surface and tile drainage nitrate loss," Journal of Environmental Quality, vol. 25, no. 2, pp. 317-324, 1996.

[20] E. J. Kladivko, J. R. Frankenberger, D. B. Jaynes, D. W. Meek, B. J. Jenkinson, and N. R. Fausey, "Nitrate leaching to subsurface drains as affected by drain spacing and changes in crop production system," Journal of Environmental Quality, vol. 33, no. 5, pp. 1803-1813, 2004.

[21] G. W. Randall, M. J. Goss et al., "Nitrate losses to surface water through subsurface, tile drainage," in Nitrogen in the Environment: Sources, Problems and Management, R. F. Follett, J. L. Hatfield et al., Eds., pp. 95-122, Elsevier Science B.V, The Netherlands, 2001.

[22] S. K. Sipp, W. D. Lembke, C. W. Boast, J. H. Peverly, M. D. Thorne, and P. N. Walker, "Water management of corn and soybeans on a claypan soil," Transactions of the American Society of Agricultural Engineers, vol. 29, no. 3, pp. 780-784, 1986.

[23] D. L. Rausch, C. J. Nelson, and J. H. Coutts, "Water management of a claypan soil," Transactions of the American Society of Agricultural Engineers, vol. 33, no. 1, pp. 111-117, 1990.

[24] P. N. Walker, M. D. Thorne, E. C. Benham, and S. K. Sipp, "Yield response of corn and soybean to irrigation and drainage on a claypan soil," Transactions of the American Society of Agricultural Engineers, vol. 25, no. 6, pp. 1617-1621, 1982.

[25] R. L. Cooper, N. R. Fausey, and J. W. Johnson, "Yield response of corn to a subirrigation/drainage management system in Northern Ohio," Journal of Production Agriculture, vol. 12, no. 1, pp. 74-77, 1999.

[26] R. L. Cooper, N. R. Fausey, J. G. Streeter et al., "Effect of water table level on the yield of soybean grown under subirrigation/ drainage," Journal of Production Agriculture, vol. 5, pp. 180184, 1992.

[27] K. A. Nelson, C. G. Meinhardt, and R. L. Smoot, "Soybean cultivar response to subsurface drainage and subirrigation in Northeast Missouri," Crop Management. In press.

[28] S. Mostaghimi, W. D. Lembke, and C. W. Boast, "Controlleddrainage/subirrigation simulation for a claypan soil," Transactions of the American Society of Agricultural Engineers, vol. 28, no. 5, pp. 1557-1563, 1985.

[29] K. A. Nelson and C. G. Meinhardt, "Soybean yield response to pyraclostrobin and drainage water management," Agronomy Journal, vol. 103, no. 5, pp. 1359-1365, 2011.

[30] K. A. Nelson, R. L. Smoot, and C. G. Meinhardt, "Soybean response to drainage and subirrigation on a claypan soil in northeast Missouri," Agronomy Journal, vol. 103, no. 4, pp. 1216-1222, 2011.

[31] W. Wiebold, H. Mason, D. Knerr, R. Hasty, T. Belt, D. Schwab et al., "Corn Missouri crop performance," Specific Report 570, University of Missouri, Columbia, Mo, USA, 2007.

[32] D. D. Buchholz, Soil Test Interpretations and Recommendations Handbook, Department of Agronomy, University of Missouri, Columbia, Mo, USA, 2004.

[33] J. Henggler, "Woodruff Irrigation Charts," University of Missouri Extension, http://agebb.missouri.edu/irrigate/woodruff/.

[34] A. J. Noellsch, P. P. Motavalli, K. A. Nelson, and N. R. Kitchen, "Corn response to conventional and slow-release nitrogen fertilizers across a claypan landscape," Agronomy Journal, vol. 101, no. 3, pp. 607-614, 2009. 
[35] K. A. Nelson, P. C. Scharf, W. E. Stevens, and B. A. Burdick, "Rescue nitrogen applications for corn," Soil Science Society of America Journal, vol. 75, no. 1, pp. 143-151, 2011.

[36] K. A. Nelson and C. G. Meinhardt, "Foliar boron and pyraclostrobin effects on corn yield," Agronomy Journal, vol. 103, no. 5, pp. 1352-1358, 2011.

[37] M. R. Paulsen, L. O. Pordesimo, M. Singh, S. W. Mbuvi, and B. Ye, "Maize starch yield calibrations with near infrared reflectance," Biosystems Engineering, vol. 85, no. 4, pp. 455460, 2003.

[38] M. R. Paulsen and M. Singh, "Calibration of a near-infrared transmission grain analyser for extractable starch in maize," Biosystems Engineering, vol. 89, no. 1, pp. 79-83, 2004.

[39] M. Singh, M. R. Paulsen, L. Tian, and H. Yao, "Site-specific study of corn protein, oil, and extractable starch variability using nit spectroscopy," Applied Engineering in Agriculture, vol. 21, no. 2, pp. 239-251, 2005.

[40] B. C. Flett, N. W. McLaren, and F. C. Wehner, "Incidence of Stenocarpella maydis ear rot of corn under crop rotation systems," Plant Disease, vol. 85, no. 1, pp. 92-94, 2001.

[41] SAS Institute, "SAS/STAT user's guide," version 9.1.2, SAS Institute, Cary, NC, USA, 2010.

[42] J. J. Wiersma, G. R. Sands, H. J. Kandel, A. K. Rendahl, C. X. Jin, and B. J. Hansen, "Responses of spring wheat and soybean to subsurface drainage in northwest Minnesota," Agronomy Journal, vol. 102, no. 5, pp. 1399-1406, 2010. 


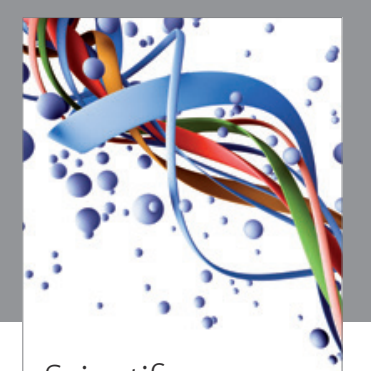

Scientifica
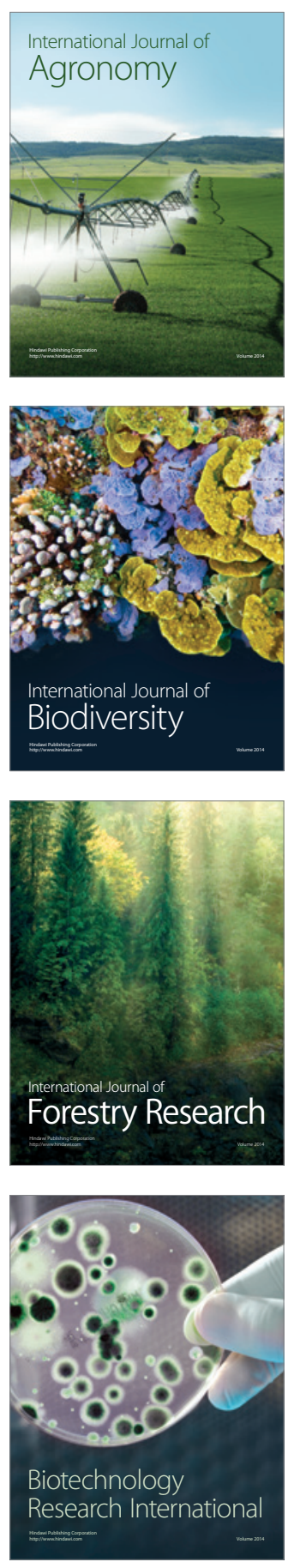
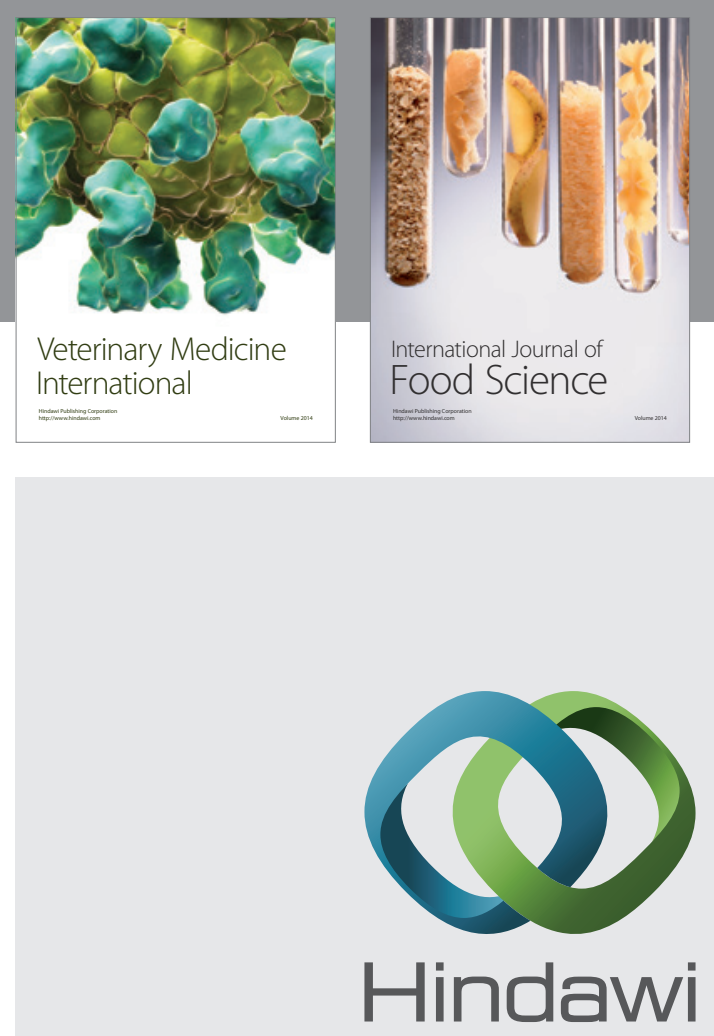

Submit your manuscripts at

http://www.hindawi.com
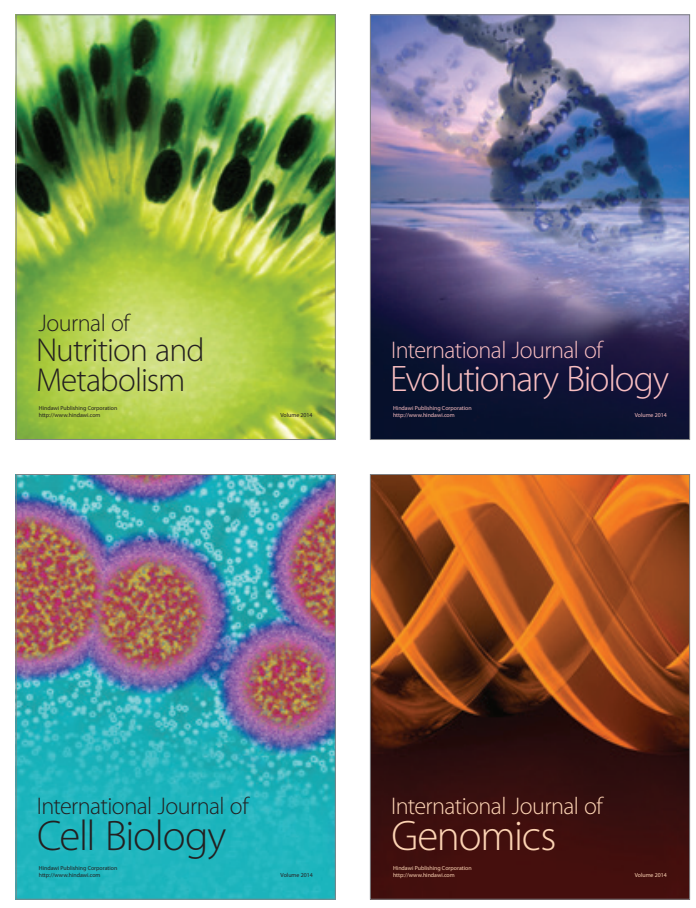
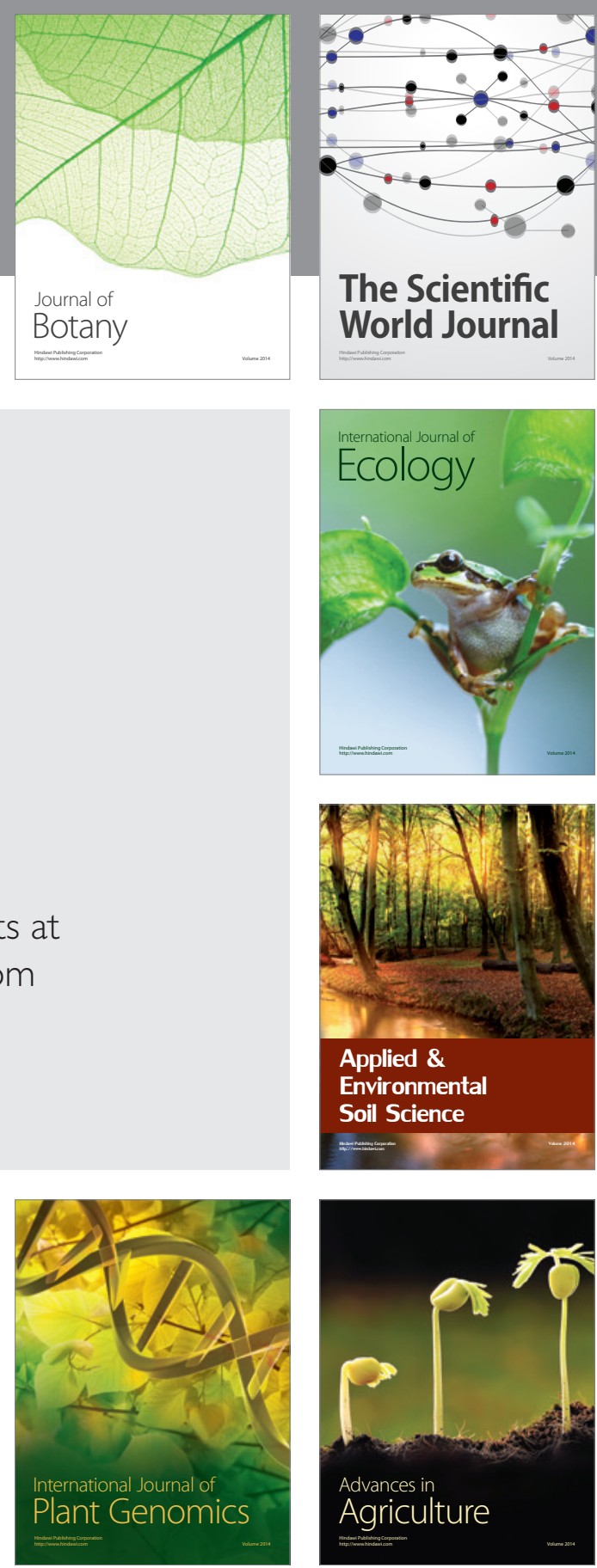

The Scientific World Journal
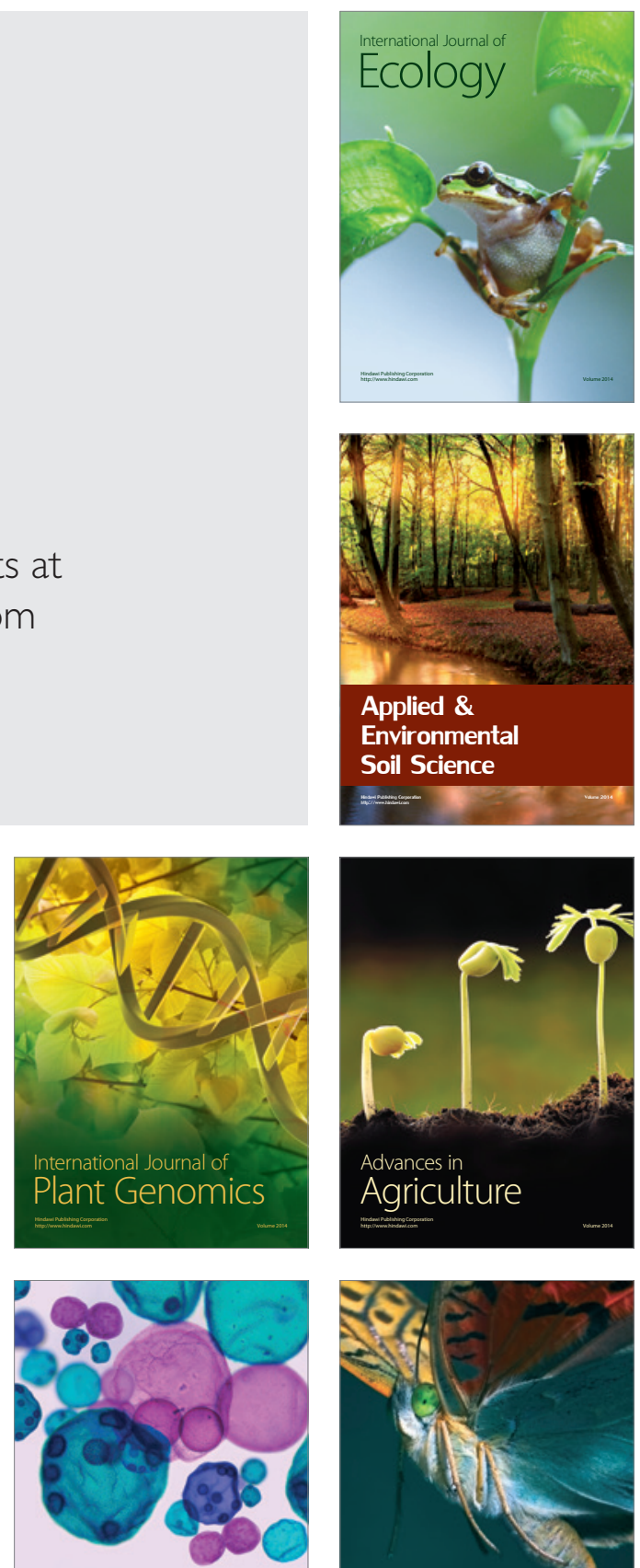

International Journal of Microbiology

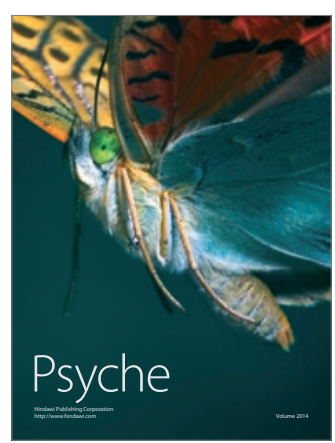

